\title{
On the magnetic topology of partially and fully convective stars
}

\author{
A. Reiners ${ }^{1, \star}$ and G. Basri ${ }^{2}$ \\ 1 Universität Göttingen, Institut für Astrophysik, Friedrich-Hund-Platz 1, 37077 Göttingen, Germany \\ e-mail: Ansgar.Reiners@phys.uni-goettingen.de \\ 2 Astronomy Department, University of California, Berkeley, CA, 94720, USA \\ e-mail: basri@berkeley.edu
}

Received 1 December 2008 / Accepted 2 January 2009

\begin{abstract}
We compare the amount of magnetic flux measured in Stokes $V$ and Stokes $I$ in a sample of early- and mid-M stars around the boundary to full convection ( M 3.5). Early-M stars possess a radiative core, mid-M stars are fully convective. While Stokes $V$ is sensitive to the net polarity of magnetic flux arising mainly from large-scale configurations, Stokes $I$ measurements can see the total mean flux. We find that in early-M dwarfs, only $\sim 6 \%$ of the total magnetic flux is detected in Stokes $V$. This ratio is more than twice as large, $\sim 14 \%$, in fully convective mid-M dwarfs. The bulk of the magnetic flux on M-dwarfs is not seen in Stokes $V$. This is presumably because magnetic flux is mainly stored in small scale components. There is also more to learn about the effect of the weak-field approximation on the accuracy of strong field detections. In our limited sample, we see evidence for a change in magnetic topology at the boundary to full convection. Fully convective stars store a 2-3 times higher fraction of their flux in fields visible to Stokes $V$. We estimate the total magnetic energy detected in Stokes $I$ and compare it to results from Stokes $V$. We find that in early-M dwarfs only $\sim 0.5 \%$ of the total magnetic energy is detected in Stokes $V$ while this fraction is $\sim 2.5 \%$ in mid-M dwarfs.
\end{abstract}

Key words. stars: late-type - stars: magnetic fields - stars: activity

\section{Introduction}

Magnetic fields are ubiquitous in cool stars. There is growing evidence that their total strength is mainly a question of rotation, and that the efficiency of magnetic field generation follows similar rules in solar-type stars and much cooler objects including planets (Christensen et al. 2009). While magnetic field generation in solar-type stars is probably most efficient near the interface layer between the radiative core and the convective envelope (e.g., Charbonneau 2005), the dynamo process in fully convective stars must be different. The transition from partially to fully convective interiors happens around a mass of $0.35 M_{\odot}$. In lower-mass stars, mean magnetic flux does not significantly differ from partially convective stars (Reiners \& Basri 2007), although they probably operate a different type of dynamo (e.g., Durney et al. 1993)

The solar magnetic field is predominantly axisymmetric and dipolar (see, e.g., Ossendrijver 2003), which we know from direct imaging of the Sun. For other stars, no direct images are available and we have to rely on indirect methods to investigate magnetic topologies. Magnetic fields are usually measured in different Stokes components via the Zeeman effect. Two main methods can be distinguished: 1) Zeeman splitting in Stokes $I$ measures the total mean magnetic flux on the star; and 2) Stokes $V$ measures effective magnetic polarization. In order to completely characterize the magnetic topology of a star, in principle all four Stokes components are necessary (e.g., Kochukhov \& Piskunov 2002), but this is observationally extremely difficult to achieve. The method of Zeeman Doppler Imaging in Stokes $V$ has been very successfull during the last years providing the first information on the magnetic structure on low-mass stars (e.g., Donati et al. 2008; Morin et al. 2008, and references therein).

^ Emmy Noether Fellow.
Using Stokes $V$, however, implies the problem that only the net polarization is visible and that probably much of the magnetic flux cancels out and is unobservable. The observation of dense time series helps resolving smaller structures but cannot entirely solve that problem. Because Stokes $I$ is sensitive to the total magnetic flux, and Stokes $V$ is sensitive to the net (large-scale) polarization, a comparison of both yields information about the amount of small scale flux that is invisible to Stokes $V$. The first question we address here is how much magnetic flux escapes detection in Stokes $V$ Doppler maps.

Recently, Morin et al. (2008) and Donati et al. (2008) used magnetic maps from Stokes $V$ to investigate magnetic topologies around the boundary to full convection. They found that the fraction of axisymmetric fields and the low- $l$ dipole modes are larger in fully convective stars than in partially convective ones. Thus, the magnetic flux visible to Stokes $V$ is more organized in fully convective stars. In this paper, we ask the question whether a higher degree of organisation in fully convective stars applies to the total magnetic flux, or to the flux visible in Stokes $V$ only.

\section{Data}

Most of the data used for our analysis are taken from the literature. Magnetic flux measurements from Stokes $V$ are taken from Donati et al. (2008) for the early-M dwarfs and from Morin et al. (2008) for mid-M dwarfs. These authors also provide rotational period and Rossby numbers for the sample stars (the latter taken from Kiraga \& Stępień 2007).

Stokes I measurements of magnetic flux are also taken from the literature, and we present two new measurements in this work. The magnetic flux of EV Lac (Gl 873) was measured by Johns-Krull \& Valenti (2000) using a detailed model of Zeeman splitting in an atomic line. This result was used by Reiners \& Basri (2007) to calibrate measurements of magnetic flux using 

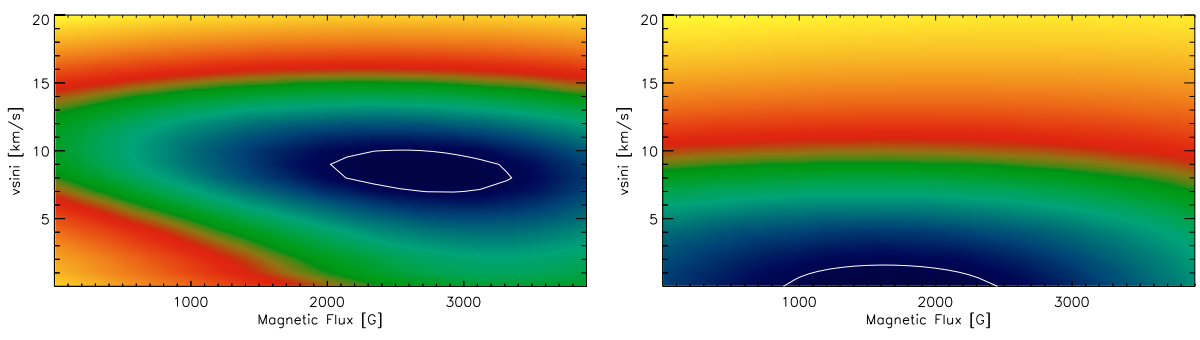

Fig. 1. $\chi^{2}$-landscapes of our model to the data of Gl 182 (left) and Gl 569A (right) as a function of projected rotation velocity $v \sin i$ and mean magnetic flux, $B f$. Different colours indicated different values of $\chi^{2}$; blue dark colours mark areas with low $\chi^{2}$ (good fit), red and yellow are regions of high $\chi^{2}$ (bad fit). The white contour is sorrounding the region where $\chi^{2}<\chi_{\min }^{2}+9$, i.e., the $3-\sigma$ region. molecular $\mathrm{FeH}$ as a tracer. Our values of $B f$ for AD Leo (G1 388) and YZ Cmi (Gl 285) are taken from Reiners \& Basri (2007). For DT Vir (Gl 494A) we found a magnetic flux measurement in Saar (1996) using atomic lines $(B=3.0 \mathrm{kG}, f=50 \%)$. Because magnetic field measurements may be affected by the choice of absorption lines used for analysis, we apply a correction factor to the $B f$ value of DT Vir based on comparison between other $B f$ measurements in Reiners \& Basri (2007) and Saar (1996); the four stars AD Leo, YZ Cmi, EV Lac, and G1 729 were investigated in both works. The magnetic flux $B f$ reported by Reiners \& Basri (2007) is systematically higher than the values reported by Saar (1996). Specifically, the results for YZ Cmi, EV Lac, and Gl 729 are consistent if the filling factor $f$ in Saar (1996) is assumed to be one. In other words, $B$ in Saar (1996) has roughly the same value as $B f$ in Reiners \& Basri (2007). Thus, we use $\left\langle B_{I}\right\rangle=3.0 \mathrm{kG}$ for DT Vir, which brings all values of $\left\langle B_{I}\right\rangle$ used here on a consistent scale. We note that an uncertainty of a factor of 2 in $\left\langle B_{I}\right\rangle$ would not influence the results of this paper.

We present additional magnetic flux measurements of two other stars in this work. Data of Gl $182(J=7.1)$ were obtained with HIRES at the W. M. Keck observatory in the same way as reported in Reiners \& Basri (2007). We used a slit-width of $1.15^{\prime \prime}$ yielding a spectral resolving power of $R \approx 31000$. The $600 \mathrm{~s}$ exposure has a SNR of about 120 at $9930 \AA$. A spectrum of CE Boo (G1 569A, $J=6.6$ ) was obtained at the Hobby-EberlyTelescope using HRS centered at $8991 \AA$. The $2^{\prime \prime}$ fibre was used providing a spectral resolving power of $R \approx 60000$. After $300 \mathrm{~s}$ a SNR of 40 was reached in the $\mathrm{FeH}$ band.

\section{New Bf measurements}

\subsection{Method}

The method we employ to measure the magnetic flux in G1 182 and CE Boo was introduced in Reiners \& Basri (2006) and after that applied to several stars (e.g., Reiners \& Basri 2007; Reiners et al. 2008). Here, we give a brief overview of the method and refer to the literature for a more detailed description.

The absorption band of $\mathrm{FeH}$ contains a forest of strong, well isolated lines of which some are sensitive to the Zeeman effect while others are not. In a relatively small spectral range, we find spectral lines of the same ro-vibrational transition that react differently to the presence of a magnetic field. The direct simulation of the Zeeman effect in $\mathrm{FeH}$ calculating polarized radiative transfer is still hampered by the lack of Landé factors. Instead, we choose a more empirical approach: we observed two spectra of early-M dwarfs for which magnetic field measurements from atomic absorption lines exist. One of them shows no signs of magnetic fields (and no activity; GJ 1002), for the other a total magnetic flux of $B f \sim 3.9 \mathrm{kG}$ was measured (Gl 873, Johns-Krull \& Valenti 2000). We apply an optical-depth scaling to the two reference spectra so that the strength of the $\mathrm{FeH}$ band matches the strength of $\mathrm{FeH}$ absorption in the target star. The shape of magnetically insensitive lines is used to fix the rotational velocity, and magnetically sensitive lines to adjust for the magnetic flux. This is done by interpolating the two template spectra (between zero magnetic flux and $B f=3.9 \mathrm{kG}$ ) in order to achieve the best fit to the target spectrum.

\subsection{Magnetic flux in GI 182 and CE Boo}

The results of our fitting procedure are shown in Fig. 1, where the goodness of fit in terms of $\chi^{2}$ is shown as a function of $B f$ and $v \sin i$. The white line surrounds the region of $\chi^{2}<\chi_{\min }^{2}+9$, i.e., the $3 \sigma$ limit (see Reiners et al. 2008, for more details). In both cases, the minimum value of the reduced $\chi^{2}$ is $\chi_{\min , v}^{2} \approx 1$. The formal results of our fitting procedure are, for Gl 182, $v \sin i=9 \pm 2 \mathrm{~km} \mathrm{~s}^{-1}, B f=2730 \pm 600 \mathrm{G}$, and for CE Boo, $v \sin i<3 \mathrm{~km} \mathrm{~s}^{-1}, B f=1750 \pm 800 \mathrm{G}(3 \sigma)$.

\section{Total flux and large-scale flux}

\subsection{Magnetic flux in Stokes $V$ and I}

We compile measurements of mean magnetic flux from Stokes $V,\left\langle B_{V}\right\rangle$, and Stokes $I,\left\langle B_{I}\right\rangle$, for the six $\mathrm{M}$ dwarfs in Table 1 together with their mass, rotational period, and Rossby number as given in Donati et al. (2008) and Morin et al. (2008). Reiners \& Basri (2007) discuss the uncertainties of magnetic flux measurements in $\mathrm{FeH}$, they found 2- $\sigma$ uncertainties on the order of $200 \mathrm{G}$ but estimate total uncertainties (including systematics) on the order of a $\mathrm{kG}$. The uncertainties we give for the two new measurements in Sect. 3.2 are 600 and $800 \mathrm{G}$ (3$\sigma)$. We adopt $800 \mathrm{G}$ as uncertainty in $\left\langle B_{I}\right\rangle$ for all stars. The ratio $\left\langle B_{V}\right\rangle /\left\langle B_{I}\right\rangle$ is given in Col. 8. The fraction of magnetic flux visible in Stokes $V$ is always less than $15 \%$ of the total flux measured in Stokes $I$. The difference between partially convective and fully convective stars is quite obvious: in partially convective stars roughly $6 \%$ of the total flux is detected in Stokes $V$ while in fully convective stars about $14 \%$ are detected.

The fraction of magnetic flux seen in Stokes $V$ (center panel of Fig. 2) never exceeds a value of $\sim 15 \%$, which means that more than $85 \%$ of the magnetic flux is invisible to magnetic flux measurements in Stokes $V$. A possible explanation is that the vast majority of the magnetic flux on M dwarfs is organized in small structures that are distributed over the stellar surface so that different polarities cancel out each other in Stokes $V$. This situation would be similar to the solar case, where the strongest magnetic fields are concentrated in spots that consist of neighbored regions of different polarity.

Another factor to be considered are the simplifications inherent to the results from Stokes $V$ used here. The technique used by Donati et al. (2008) and Morin et al. (2008) makes a few important assumptions. First, the "weak-field approximation" is used (Semel 1989). Donati \& Brown (1997) estimate that the weakfield approximation becomes incorrect at fields on the order of $1.2 \mathrm{kG}$, but they find it is still adequate to fields up to $5 \mathrm{kG}$ (see also Wade et al. 2000). The weak-field approximation becomes 


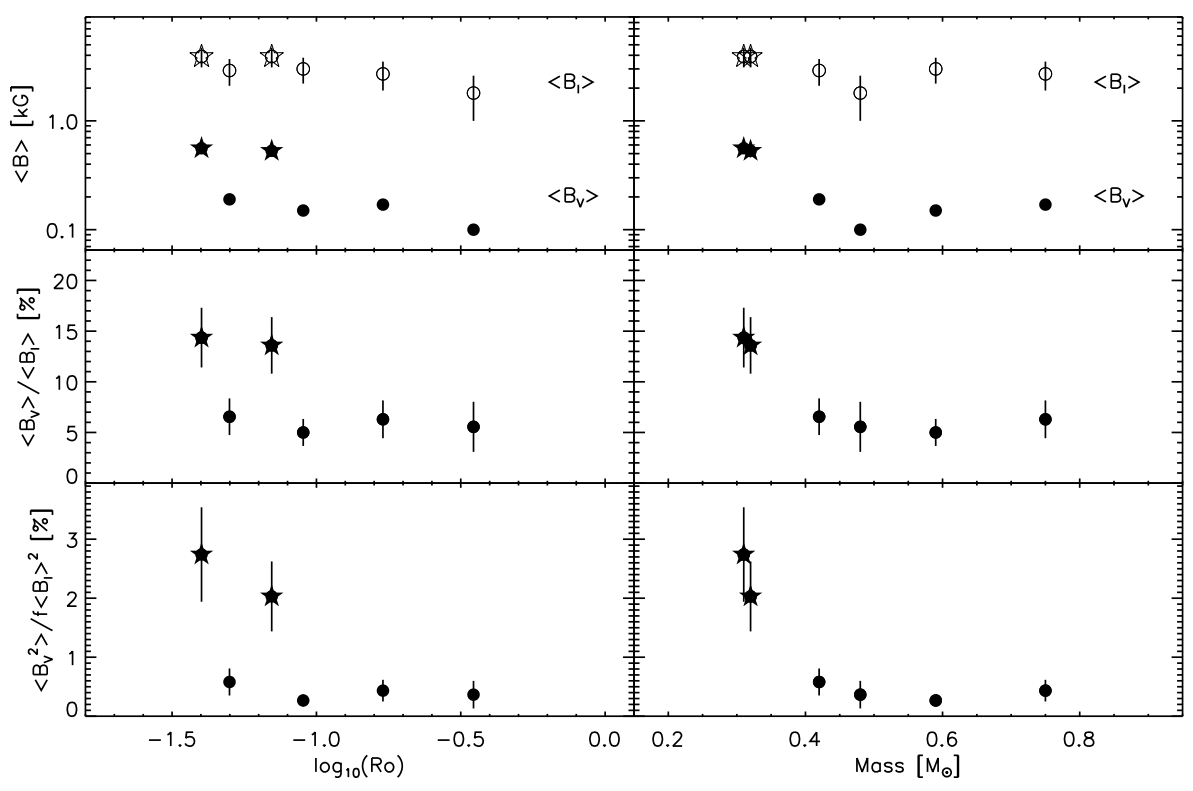

Fig. 2. Top panel: mean magnetic field measurements from Stokes $I$ (open symbols) and Stokes $V$ (filled symbols). Center (bottom) panel: ratio of large-scale magnetic flux (energy) to total magnetic flux (energy). Left and right panels show these values as a function of Rossby number and mass, respectively. Symbols distinguish between fully convective (stars) and partially convective (circles) stars.

Table 1. Properties of the sample stars. Rotation period and results from Stokes $V$ measurements are from Donati et al. (2008) and Morin et al. (2008). Rossby numbers are from Kiraga \& Stępień (2007). Sources of Stokes $I$ measurements are given in the table. The total magnetic energy is estimated from total magnetic flux using a scaling that involves a factor $f$. We use $f=1.25$ (see text).

\begin{tabular}{|c|c|c|c|c|c|c|c|c|c|c|}
\hline Name & Other & $\begin{array}{l}\text { Mass } \\
{\left[M_{\odot}\right]}\end{array}$ & $\begin{array}{l}P_{\text {rot }} \\
{[\mathrm{d}]} \\
\end{array}$ & $R o$ & $\begin{array}{l}\left\langle B_{V}\right\rangle \\
{[\mathrm{kG}]} \\
\end{array}$ & $\begin{array}{l}\left\langle B_{I}\right\rangle \\
{[\mathrm{kG}]} \\
\end{array}$ & $\begin{array}{l}\frac{\left\langle B_{V}\right\rangle}{\left\langle\left\langle V_{I}\right\rangle\right.} \\
{[\%]} \\
\end{array}$ & $\begin{array}{c}\left\langle B_{V}^{2}\right\rangle \\
{\left[\mathrm{kG}^{2}\right]} \\
\end{array}$ & $\begin{array}{c}f \cdot\left\langle B_{I}\right\rangle^{2} \\
{\left[\mathrm{kG}^{2}\right]}\end{array}$ & $\begin{array}{c}\frac{\left\langle B_{V}^{2}\right\rangle}{f \cdot\left\langle B_{I}\right\rangle^{2}} \\
{[\%]} \\
\end{array}$ \\
\hline Gl 182 & & 0.75 & 4.35 & 0.17 & 0.17 & $2.5^{a} \pm 0.8$ & $6.3 \pm 1.9$ & 0.04 & $9.1 \pm 3.9$ & $0.4 \pm 0.2$ \\
\hline Gl 494A & DT Vir & 0.59 & 2.85 & 0.09 & 0.15 & $3.0^{b} \pm 0.8$ & $5.0 \pm 1.3$ & 0.03 & $11.3 \pm 4.3$ & $0.3 \pm 0.1$ \\
\hline Gl 569A & CE Boo & 0.48 & 14.7 & 0.35 & 0.10 & $1.8^{a} \pm 0.8$ & $5.6 \pm 2.5$ & 0.01 & $4.1 \pm 2.6$ & $0.4 \pm 0.2$ \\
\hline Gl 388 & AD Leo & 0.42 & 2.24 & 0.05 & 0.19 & $2.9^{c} \pm 0.8$ & $6.6 \pm 1.8$ & 0.06 & $10.5 \pm 4.1$ & $0.6 \pm 0.2$ \\
\hline G1 873 & L La & 0.32 & . & 0.07 & 0.53 & $3.9^{d} \pm 0.8$ & $13.6 \pm 2.8$ & 0.39 & $19.0 \pm 5.6$ & $2.0 \pm 0.6$ \\
\hline Gl 285 & YZ Cmi & 0.31 & 2.77 & 0.04 & 0.56 & $\gtrsim 3.9^{c} \pm 0.8$ & $\lessgtr 14.4 \pm 3.0$ & 0.52 & $\gtrsim 19.0 \pm 5.6$ & $\lesssim 2.7 \pm 0.8$ \\
\hline
\end{tabular}

${ }^{a}$ This work; ${ }^{b}$ Saar (1996), see text; ${ }^{c}$ Reiners \& Basri (2007); ${ }^{d}$ Johns-Krull \& Valenti (2000).

particularly relevant together with the second simplification, the use of a Least-Square-Deconvolution technique (Donati et al. 1997; Donati \& Collier Cameron 1997; Wade et al. 2000). In the weak-field approximation, different Landé factors enter the profile as a scaling parameter that can be accounted for in a mask (like the line-depth). This makes LSD applicable to the weakfield case. The detectability of strong fields may be hampered because very strong Zeeman signals cannot be fully taken into account. It is still an open question whether fields on the order of $2-3 \mathrm{kG}$ can be accurately reconstructed with this method. The only way to address this would be to compute stellar surfaces with real radiative transfer in all lines.

For now, we can only speculate that the Stokes $V$ maps used for this analysis may systematically miss magnetic fields stronger than a few $\mathrm{kG}$. This opens a rather interesting option for the explanation of the discontinuity at the boundary to full convection: if, in contrast to partially convective stars, fully convective stars have magnetic flux less concentrated in small areas of strong magnetic fields, but more evenly distributed in large areas of weaker fields, more of the flux may be detectable in fully convective stars. This is an interesting alternative that could imply a weaker degree of organization in fully convective $\mathrm{M}$ dwarfs rather than a stronger large-scale component. Unfortunately, this alternative is difficult to test with current instrumentation. So far, the mentioned assumptions are necessary to detect the subtle signatures of stellar magnetic fields on Stokes $V$.

\subsection{Trends with mass and Rossby number}

The mean magnetic flux and the ratio between magnetic flux observed in Stokes $V$ and Stokes $I$ are plotted in the upper two panels of Fig. 2. Left and right panels show them as a function of Rossby number and mass, respectively. Uncertainties in $\left\langle B_{I}\right\rangle$ are estimated to be $800 \mathrm{G}$ in all stars, which is mainly due to systematic effects. No error estimates are available for $\left\langle B_{V}\right\rangle$.

The upper left panel of Fig. 2 shows the rotation-magnetic flux relation: total magnetic flux, $\left\langle B_{I}\right\rangle$, grows with smaller $R o$ in the regime $\log R o>-1$ and saturates at lower Rossby number (cp. Reiners et al. 2008). Total magnetic flux may also depend on mass with higher $\left\langle B_{I}\right\rangle$ at lower masses, but mass and Rossby number to some extent are degenerate because of the large convective overturn times in low mass stars (at lower mass, saturation occurs at lower rotation rate). On the other hand, the largescale flux, $\left\langle B_{V}\right\rangle$, shows a very clear dependence on stellar mass. A dependence of $\left\langle B_{V}\right\rangle$ on Rossby number cannot be excluded.

The ratio between small-scale and total magnetic flux shows a discontinuity at the boundary where stars are thought to become completely convective: while $\left\langle B_{V}\right\rangle /\left\langle B_{I}\right\rangle$ is about $6 \%$ in early-M dwarfs with masses above $0.35 M_{\odot}$, it is about $14 \%$ in mid-M dwarfs with $M<0.35 M_{\odot}$

It is notoriously difficult to disentangle effects in Rossby number and stellar mass because low-mass $M$ dwarfs have Rossby numbers generally lower than higher mass $M$ dwarfs. Here, AD Leo is substantially more massive than EV Lac and 
YZ Cmi. On the other hand, AD Leo has a Rossby number lower than EV Lac due to AD Leo's higher rotation rate. Regardless of rotation, however, AD Leo shows substantially less small-scale magnetic flux a factor of 2-3 lower than in EV Lac (while its total magnetic flux is only $\sim 25 \%$ less than in EV Lac).

\section{Magnetic energy}

Sometimes it is also interesting to investigate magnetism in terms of magnetic energy, which is proportional to the magnetic flux squared. From Doppler tomography, the magnetic energy measured in Stokes $V,\left\langle B_{V}^{2}\right\rangle$, is reported (Donati et al. 2008; Morin et al. 2008) and given in Col. 9 of Table 1. From Stokes $I$, detailed information about the flux distribution on the stellar surface is usually not given so that $\left\langle B_{I}^{2}\right\rangle$ is not available.

In order to calculate the ratio between the magnetic energies measured in Stokes $V$ and Stokes $I$, i.e., the ratio between the large-scale magnetic energy to the total magnetic energy, we estimate from the mean total magnetic flux, $\left\langle B_{I}\right\rangle$, the mean total magnetic energy, $\left\langle B_{I}^{2}\right\rangle$, which is generally not identical to the square of the total mean flux, $\left\langle B_{I}\right\rangle^{2}$. The difference between $\left\langle B_{I}^{2}\right\rangle$ and $\left\langle B_{I}\right\rangle^{2}$ is approximately equal to the Variance of the magnetic flux distribution. In case of a completely uniform distribution $(\sigma=0)$, the two are identical. The distribution can be characterized by the standard deviation, which is the square root of the Variance, or

$\sigma^{2}=\operatorname{Var}(B) \approx\left\langle B^{2}\right\rangle-\langle B\rangle^{2}$.

For Stokes $V$, we can calculate the Variance of the magnetic flux distribution, $\left\langle B_{V}^{2}\right\rangle-\left\langle B_{V}\right\rangle^{2}$, and find that $\sigma_{V} \sim k_{V}\left\langle B_{V}\right\rangle$ with $k_{V}$ ranging from 0.61 to 0.83 with a mean of 0.69 . From Eq. (1) we derive $\left\langle B_{V}^{2}\right\rangle \approx 1.5\left\langle B_{V}\right\rangle^{2}$. The distribution of magnetic flux measured in Stokes $I$ can be expected to be more evenly distributed than the flux detected in Stokes $V$, because $85-95 \%$ of it is not seen in Stokes $V$. It is probably distributed in rather small entities. Thus, the Variance in magnetic field distribution seen in $\left\langle B_{I}\right\rangle$ is probably smaller than in $\left\langle B_{V}\right\rangle$. We make the assumption that the Variance of the total flux distribution is $\sigma_{I}=0.5\left\langle B_{I}\right\rangle\left(k=0.5\right.$ instead of 0.7 as for $\left.\left\langle B_{V}\right\rangle\right)$, i.e., $f \approx 1.25$ in $\left\langle B_{I}^{2}\right\rangle=f\left\langle B_{I}\right\rangle^{2}$. The exact choice of $f=[1.0 \ldots 1.5]$ has no effect on our results. In the last column of Table 1, we give the ratio between the magnetic energy detected in Stokes $V$ and the estimated magnetic energy seen in Stokes $I$.

The lower panel of Fig. 2 shows the behavior of magnetic energy with Rossby number and mass. Uncertainties in the ratio of magnetic energies include the uncertainty in the scaling factor $f$, which is regarded as $f=1.25 \pm 0.25$ (see above). As expected, magnetic energy goes as magnetic flux. As a consequence of magnetic energy being essentially the magnetic flux squared, the discontinuity at $M \approx 0.35 M_{\odot}$ is even more pronounced.

\section{Summary}

We compared the results of magnetic flux measurements carried out in Stokes $V$ and Stokes $I$ in order to characterize the magnetic field topology of early- and mid-M dwarfs. Early-M dwarfs $(<\mathrm{M} 3.5)$ are believed to harbor a radiative core while later stars are probably fully convective. The Stokes $V$ results are mainly sensitive to large scale fields because signal from magnetic areas of opposite polarity cancel out each other. Simplifications (mainly the weak-field approximation) may also have some influence on the detectability of strong field components. Stokes $I$ is an indicator of the total mean flux because all magnetic fields are seen regardless of polarity and organization.

Our sample comprises four partially convective and two fully convective stars assuming that the boundary to full convection is at $M=0.35 M_{\odot}$. The ratio between magnetic flux seen in Stokes $V$ and Stokes $I$ is around $6 \%$ for partially convective stars and $14 \%$ for fully convective stars. The fraction of magnetic energy stored in magnetic fields visible to Stokes $V$ is around $0.5 \%$ of the total flux in early-M dwarfs and $2.5 \%$ in mid-M dwarfs. Our two main results are the following:

1. In M dwarfs, more than $85 \%$ (96\%) of the magnetic flux (energy) is stored in magnetic fields that are invisible to Stokes $V$.

2. The fraction of the total magnetic flux that is detected in Stokes $V$ shows a remarkable jump at the boundary to full convection. In our limited sample, the fully convective stars store about 2-3 times more magnetic flux (5 times more magnetic energy) in large scale fields visible to Stokes $V$ than partially convective M-dwarfs do.

Our results partly confirm those of Donati et al. (2008); the magnetic topologies abruptly change at the full convection boundary. Donati et al. (2008) show that the magnetic energy stored in axisymmetric configurations is higher in fully convective stars than in partially convective ones. Their results were based solely on Stokes $V$ measurements. In this work we showed that this trend also applies to the fraction of large-scale to total magnetic energy. The small fraction of flux and energy detected in Stokes $V$ shows the importance of other Stokes components for a full description of a star's magnetic topology. This continues to be a challenging task for the future.

Acknowledgements. Some of the data presented herein were obtained at the W. M. Keck Observatory, which is operated as a scientific partnership among the California Institute of Technology, the University of California and the National Aeronautics and Space Administration. The Observatory was made possible by the generous financial support of the W. M. Keck Foundation.The HobbyEberly Telescope (HET) is a joint project of the University of Texas at Austin, the Pennsylvania State University, Stanford University, Ludwig-MaximiliansUniversität München, and Georg-August-Universität Göttingen. The HET is named in honor of its principal benefactors, William P. Hobby and Robert E. Eberly. We thank the referee, Michel Auriere, for a thorough and very constructive report. A.R. acknowledges research funding from the DFG as an Emmy Noether fellow under RE 1664/4-1. G.B. acknowledges support from the NSF through grant AST-0606748.

\section{References}

Charbonneau, P. 2005, Living Rev. Sol. Phys., 2, 2

Christensen, U., Holzwarth, V., \& Reiners, A. 2009, Nature, 457, 167

Donati, J.-F., \& Brown, S. F. 1997, A\&A, 326, 1135

Donati, J. F., \& Collier Cameron, A. 1997, MNRAS, 291, 1

Donati, J.-F., Semel, M., Carter, B. D., Rees, C. E., \& Cameron, A. C. 1997, MNRAS, 291, 658

Donati, J.-F., Morin, J., Petit, P., et al. 2008, MNRAS, 390, 545

Durney, B. R., de Young, D. S., \& Roxburgh, I. W. 1993, Sol. Phys., 145, 207

Johns-Krull, C., \& Valenti, J.A. 2000, ASPC, 198, 371

Kiraga, M., \& Stȩpień, K. 2007, AcA, 57, 149

Morin, J., Donati, J.-F., Petit, P., et al. 2008, MNRAS, 390, 567

Ossendrijver, M. 2003, A\&AR, 11, 287

Kochukhov, O., \& Piskunov, N. 2002, A\&A, 381, 736

Reiners, A., \& Basri, G. 2006, ApJ, 644, 497

Reiners, A., \& Basri, G. 2007, ApJ, 656, 1121

Reiners, A., Basri, G., \& Browning, M. 2008, ApJ, in press

Saar, S. H. 1996, IAU Symp., 176, 237

Semel, M. 1989, A\&A, 225, 456

Wade, G. A., Donati, J.-F., Landstreet, J. D., \& Shorlin, S.L.S. 2000, MNRAS, 313,823 